\title{
Control Access Point of Devices for Delay Reduction in WBAN Systems with CSMA/CA
}

\author{
Akinobu Nemoto1, Pham Thanh Hiep², Ryuji Kohno² \\ ${ }^{1}$ Department of Medical Informatics, Yokohama City University, Yokohama, Japan \\ ${ }^{2}$ Division of Physics, Electrical and Computer Engineering, Yokohama National University, Yokohama, Japan \\ Email: anemoto@med.yokohama-cu.ac.jp, hiep@ynu.ac.jp, kohno@ynu.ac.jp
}

Received 10 January 2015; accepted 26 January 2015; published 29 January 2015

Copyright (C) 2015 by authors and Scientific Research Publishing Inc.

This work is licensed under the Creative Commons Attribution International License (CC BY). http://creativecommons.org/licenses/by/4.0/

(c) (i) Dpen Access

\begin{abstract}
Due to the gathering of sickrooms and consultation rooms in almost all hospitals, the performance of wireless devices system is deteriorated by the increase of collision probability and waiting time. In order to improve the performance of wireless devices system, relay is added to control the access point and then the access of devices is distributed. The concentration of access point is avoided and then the performance of system is expected to be improved. The discrete time Markov chain (DTMC) is proposed to calculate the access probability of devices in a duration time slot. The collision probability, throughput, delay, bandwidth and so on are theoretically calculated based on the standard IEEE802.15.6 and the performance of the system with and without relay is compared. The numerical result indicates that the performance of the system with control access point is higher than that of the system without control access point when the number of devices and/or packet arrive rate are high. However, the system with control access point is more complicated. It is the trade-off between the performance and the complication.
\end{abstract}

\section{Keywords}

Standard IEEE802.15.6, Discrete Time Markov Chain Method, Control Access Point, Bandwidth Efficiency, Delay

\section{Introduction}

\subsection{The Problem of WLAN in Hospitals}

In almost hospitals, sickrooms and consultation rooms are respectively gathered at one place for convenience of patients. It may be good for patients and hospital sites, however, on the view point of wireless system, there is a problem. Since medical devices access the wireless local area network (WLAN) base station via wireless chan- 
nel, the collision when more than one devices access the channel in the same time, will occurs depending on the number of devices and the number of data packets that be generated by every device in one second. Moreover, a lot of devices access the WLAN base station that is close to the consultation rooms (Wireless LAN 2 in Figure 1), whereas a few devices access the WLAN base station that is far from consultation rooms (Wireless LAN 1). The access of devices concentrates at Wireless LAN 2, consequently, the probability of collision increases, and then the throughput decreases, the delay increase. As a result the bandwidth efficiency decreases.

\subsection{Aims and Motivations}

Since many body functions are traditionally monitored and separated by a considerable period of time, it is hard for doctors to know what is really happening. This is the reason why the monitoring of movement and all body functions in daily life are essential. The delay of patients' data as well as the collision of data packets may let doctors misunderstand and information data be lost by timeout. In order to decrease the delay and increase the throughput, the relay can be set to avoid the concentration of WLAN base station. As shown in Figure 1), some devices assess the wireless LAN 1 via the relay, therefore, the number of devices that access the wireless LAN 2 is reduced, and then the bandwidth efficiency is expected to be higher. However, the delay due to signal processing at relay should be considered. At scheme 1, all devices access the wireless LAN 2, whereas at scheme 2, the relay is set and devices access the channel via either wireless LAN 1 or 2. The performance of both schemes 1 and 2 is mathematically analyzed base on standard IEEE802.15.6. The throughput, delay and bandwidth efficiency of both schemes are numerically compared.

\subsection{Related Works}

According to an emergency of wireless body area network (WBAN), the standard IEEE802.15.6 was established in Feb. 2012 [1]. An overview of the standard and performance analyses of WBAN based on bandwidth efficiency and delay were represented in [2]-[4]. In these papers, however, the WBAN is assumed to consists of only one device that keeps transmitting a data packet. Packet arrival rates and collisions due to transmission of multiple devices in the same time weren’t considered. On the other hand, a Physical layer (PHY), Media Access Control (MAC) layer and network layer of WBAN were researched in [5] [6]. Furthermore, the control on MAC layer was analyzed to improve the performance of WBANs [7] [8]. The transmission of implanted devices was considered under conditions of low transmit power and low harmful influence on a human body [9] [10]. The performance of WBANs that has multiple devices and multiple user priorities were analyzed in both saturation [11] [13] [14] and non-saturation [12]. Additionally, WBANs were analyzed in further detail when a superframe with beacon mode and an access phases length were taken into consideration in [13] [14], respectively. However, efficiencies of number of devices, packet arrival rates, packet sizes, etc. on the throughput of each device and the total throughput, the delay and the bandwidth efficiency of system hasn't been discussed.

\subsection{Organization of the Paper}

The rest of paper is organized as follows. We introduce a brief of PHY and MAC layers of standard IEEE802.15.6

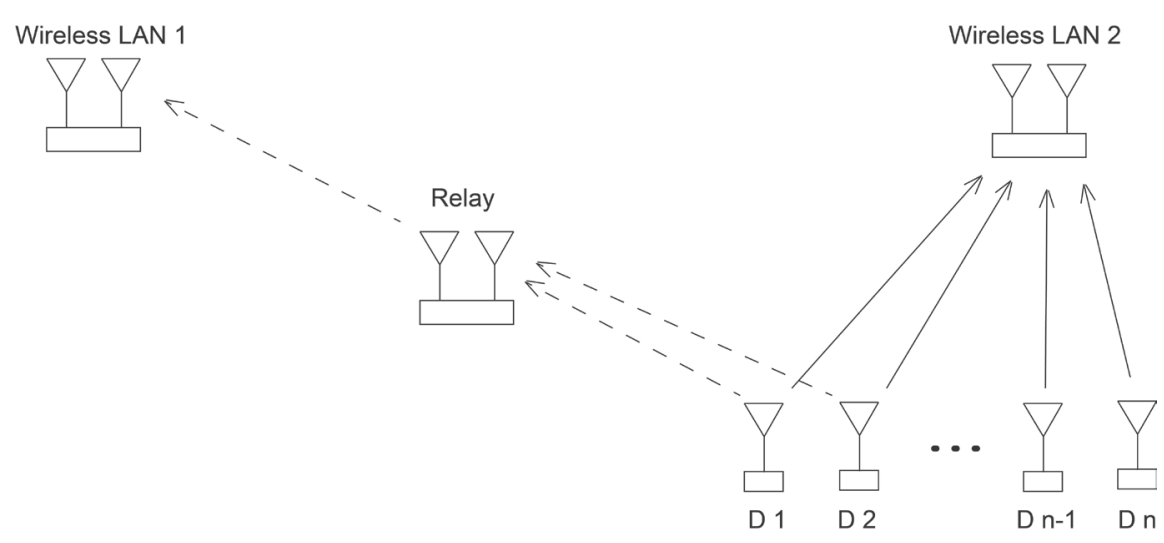

Figure 1. The wireless LAN system in a hospital. 
in Section 2. The discrete time Markov chain is proposed and then the performance of both schemes 1 and 2 with CSMA/CA is analyzed in Section 3. The numerical evaluation of both schemes is described and compared in Section 4. Finally, Section 5 concludes the paper.

\section{Brief of Standard IEEE802.15.6}

A brief of the standard that related to our research is described in this section. The further detail of standard can be found in [1] [2].

\subsection{PHY Layer}

The IEEE802.15.6 defines three different PHYs, i.e., human body communication (HBC), narrowband (NB) and ultra wideband (UWB). Furthermore, the NB is divided in several frequency bands and a data rate, symbol rate, etc. of every frequency band are different. We analyze the system in $2400 \mathrm{MHz}-2483.5 \mathrm{MHz}$ band as an example, the analysis in different frequency band is similar. The physical protocol data unit (PPDU) of NB PHY is described in Figure 2. Components of PPDU are fixed, excepted the payload. Parameters of PHY layer are summarized in Table 1.

\subsection{MAC Layer}

The algorithm of CSMA/CA based on IEEE802.15.6 is described as follows. All devices set their backoff counter

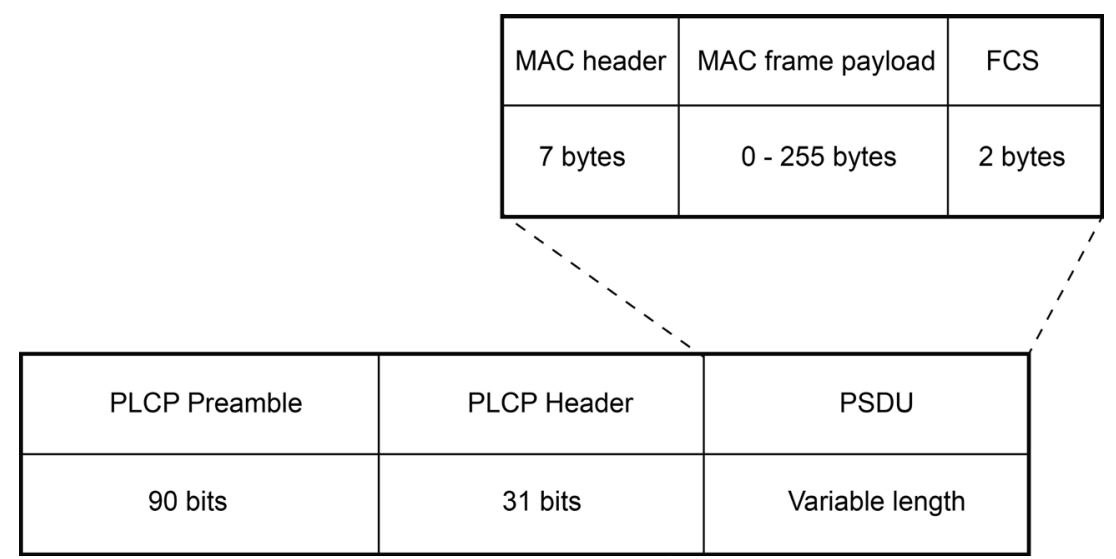

Figure 2. PPDU of NB PHY.

Table 1. Parameters of PHY layer.

\begin{tabular}{cc}
\hline Frequency band $[\mathrm{MHz}]$ & $2400-2483.5$ \\
Packet component & PSDU \\
Modulation DBPSK & DBPSK \\
Symbol rate $R s[\mathrm{ksps}]$ & 600 \\
Data rate $R h d r[\mathrm{kbps}]$ & 242.9 \\
Clear channel assessment [bits] & 63 \\
MAC header [bits] & 56 \\
MAC footer [bits] & 16 \\
CSMA slot time Ts $[\mu \mathrm{s}]$ & 125 \\
Short interframe spacing time $T_{p S I F s}[\mu \mathrm{s}]$ & 75 \\
Preamble [bits] & 88 \\
Propagation delay $\alpha[\mu \mathrm{s}]$ & 1 \\
\hline
\end{tabular}


to a random integer number uniformly distributed over the interval $[1, W]$, where $W$ is a contention window within $\left(W_{\min } ; W_{\max }\right)$. The value of $W_{\min }$ and $W_{\max }$ varies depending on the user priorities (UPs). However, in this paper, the UP of all devices is assumed to be the same as zero-th UP. The extension for multiple UPs is straightforward.

As shown in Figure 3, a device starts decrementing its back off counter by one for each idle CSMA slot. When the back off counter reaches zero, the device transmits its packet. Once the channel is busy because of transmission of another device, the device locks the back off counter until the channel is idle. The transmission is failed if the device fails to receive an acknowledgement (ACK) due to a collision or being unable to decode. The $W$ is doubled for even number of failures until it reaches $W_{\max }$. The maximum number of back off stages is bound by a retry limit $\mathrm{m}$. Once the number of retries exceeds the predefined retry limit $\mathrm{m}$, the packet is discarded. When the transmission is successful, the $W$ is set to $W_{\max }$. The $\mathrm{W}$ of zero-th UP is represented in Table 2.

\section{Performance Analysis of WBANs}

\subsection{Discrete Time Markov Chain}

At first, the performance of scheme 1 is analyzed. The scheme 1 consists of a single base station, the wireless LAN 2, and n devices in a star topology, $D 1, D 2, \cdots, D$ n (Figure 1). All devices can access the wireless LAN 2 directly, however, the wireless LAN 1 is out of them range. The discrete time Markov chain (DTMC) is proposed to calculate the access probability of each device in every time slot. The proposal DTMC of device $i$ with empty state is described in Figure 4 and notations used in this section are listed in Table 3. A packet arrival rate of all devices is assumed the same and denoted by $\lambda$. Hence, $\rho=1-\mathrm{e}^{-\lambda T s}$, where e denotes the Napier's constant, denotes the probability that the device has a packet to transmit in duration time of Ts. The transmission failed probability and the idle probability of device I are respectively expressed as

$$
\begin{aligned}
P_{i, \text { fail }}= & P_{i, \text { col }}+\mathrm{PER}_{i}, \\
P_{i, \text { idle }}= & \frac{\prod_{k=1}^{n}\left(1-\tau_{k}\right)}{1-\tau_{i}},
\end{aligned}
$$

here $P_{i, \mathrm{col}}=1-\prod_{k \neq i}^{n}\left(1-\tau_{k}\right)$. The state transmission probabilities of DTMC method are represented as follows.

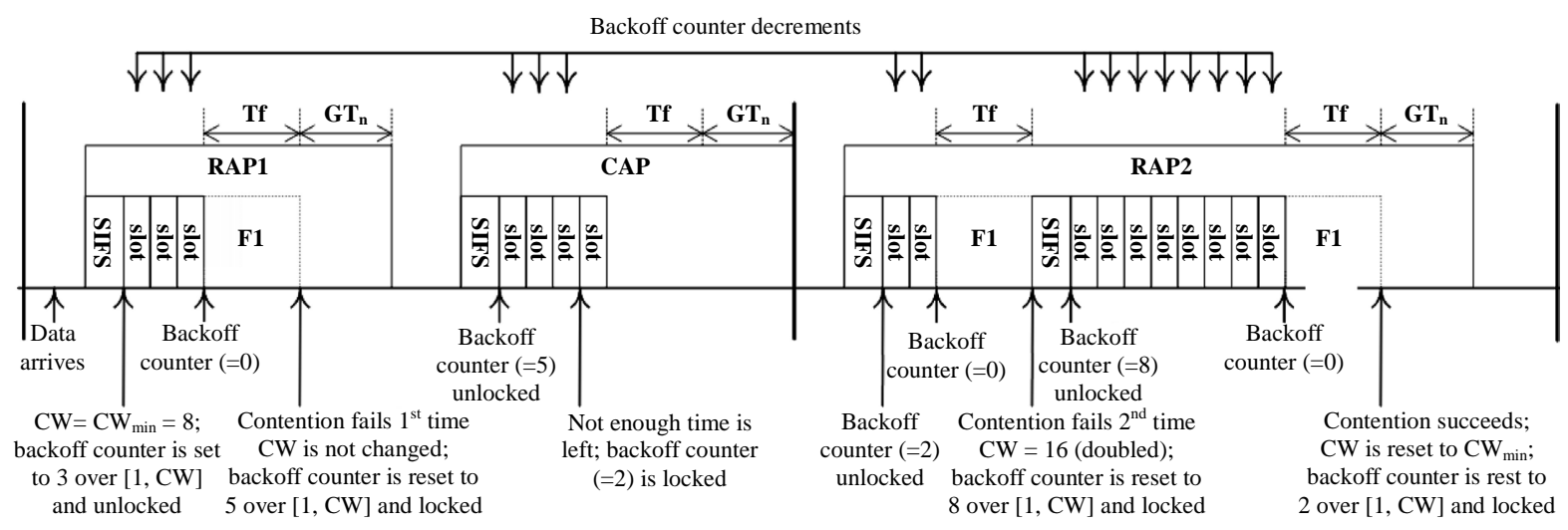

Figure 3. An example of operation of CSMA/CA and relationships of time durations.

Table 2. Contention window for every UP.

\begin{tabular}{ccccc} 
Table 2. Contention window for every UP. & & \\
\hline Number of retransmissions & 0 & 1 & 2 & 3 \\
W & 16 & 16 & 32 & 32 \\
\hline
\end{tabular}




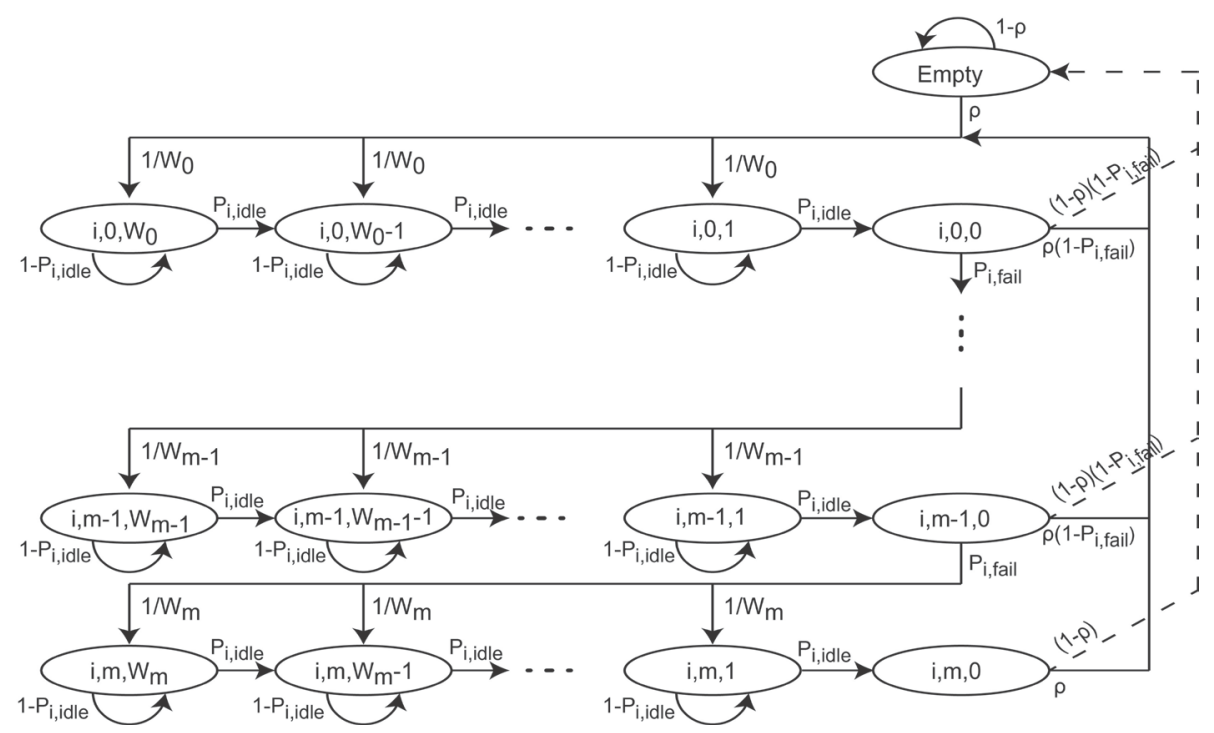

Figure 4. Algorithm of DTMC method.

\section{Table 3. Explanation of notations.}

\begin{tabular}{cl}
\hline Notation & \\
\hline$\lambda$ & Packet arrival rate during a unit time \\
$\rho$ & Packet arrival rate during a slot time \\
$m$ & Packet retry limit \\
$n$ & Total number of devices \\
$x$ & Payload size \\
Total $x$ & Total data \\
$T_{i}$ & Access probability during a slot time \\
$b_{i, k, j}$ & Stationary distribution with backoff stage $k$, backoff counter $j$ \\
$P_{i, \text { idle }}$ & Channel idle probability \\
$P_{i, \text { fail }}$ & Transmission failed probability \\
$P_{i ; \text { col }}$ & Collision probability \\
PER $_{i}$ & Packet error rate \\
$W_{k}$ & Contention window of $k$ backoff stage \\
\hline
\end{tabular}

$\operatorname{Pr}\{i, k, j \mid i, k, j+1\}=P_{i, \text { idle }}$, for $k \in[0, m], j \in\left[0, W_{k}\right]$,

$\operatorname{Pr}\{i, k, j \mid i, k-1,0\}=\frac{P_{i, \text { fail }}}{W_{k}}$, for $k \in[1, m], j \in\left[1, W_{k}\right]$,

$\operatorname{Pr}\{i, k, j \mid i, k, j\}=1-P_{i, \text { idle }}$, for $k \in[0, m], j \in\left[1, W_{k}\right]$,

$\operatorname{Pr}\{i, 0, j \mid i, m, 0\}=\frac{\rho}{W_{0}}$, for $j \in\left[1, W_{0}\right]$,

$\operatorname{Pr}\{i, 0, j \mid$ empty $\}=\frac{\rho}{W_{0}}$, for $j \in\left[1, W_{0}\right]$,

$\operatorname{Pr}\{$ empty $\mid i, k, 0\}=(1-\rho)\left(1-P_{i, \text { fail }}\right)$, for $k \in[0, m-1]$,

$\operatorname{Pr}\{$ empty $\mid i, m, 0\}=1-\rho$,

$\operatorname{Pr}\{$ empty $\mid$ empty $\}=1-\rho$. 
As shown in Figure 4, we have

$$
\sum_{k=0}^{m} \sum_{j=0}^{W_{k}} b_{i, k, j}+b_{\text {empty }}=1
$$

Moreover, the stationary distribution can be calculated by using the state transition probability.

$$
\begin{aligned}
& \sum_{k=1}^{m} b_{i, k, 0}=\frac{P_{i, \text { fail }}\left(1-P_{i, \text { fail }}^{m}\right)}{1-P_{i, \text { fail }}} b_{i, 0,0}, \\
& \sum_{j=0}^{W_{0}} b_{i, 0, j}=\frac{W_{0}+1}{2 P_{i, \text { dlle }}} b_{i, 0,0}, \\
& b_{\text {empty }}=\frac{1-\rho}{\rho} b_{i, 0,0} .
\end{aligned}
$$

From above equations, the $b_{i, 0,0}$ can be described as a function of $P_{i, \text { idle }}, P_{i, \text { fail }}, \rho, W_{k}$ and $T_{i}$, Furthermore, the access probability of every device can be calculated by solving $n$ equations.

$$
\tau_{i}=\sum_{k=0}^{m} b_{i, k, 0}=\frac{1-P_{i, \text { fail }}^{m+1}}{1-P_{i, \text { fail }}} b_{i, 0,0}
$$

\subsection{System Throughput}

The probability in which at least one device is sending a packet is called as transmission probability, $P_{\text {tran }}$.

$$
P_{\text {tran }}=1-\prod_{j=1}^{n}\left(1-\tau_{j}\right)
$$

The successful probability of device $i$ means that only device $i$ is transmitting on the medium under condition on the fact that at least one device is transmitting and is represented by $P_{i, \text { suc }}$. In addition, the coordinator can decode the packet correctly.

$$
P_{i, \text { suc }}=\frac{\tau_{i} \prod_{j=1}^{n}\left(1-\tau_{j}\right)}{\left(1-\tau_{i}\right) P_{\text {tran }}}\left(1-\mathrm{PER}_{i}\right)
$$

Let $P_{\text {suc }}=\sum_{i=1}^{n} P_{i, \text { suc }}$ denote the total successful probability of all devices. Once the transmission is successful, the device receives a ACK packet with no payload from the coordinator, whereas the device receives NACK packet or nothing after the timing to receive the ACK packet if the transmitted packet is collided or unable to decode. Consequently, the duration time to transmit a packet successfully, $T$, is assumed to equal to the duration time of failed transmission, hereafter $T$ is called as the successful transmission time. The successful transmission time is the total duration time to transmit a packet, includes the duration time to transmit a data packet $\left(T_{\text {data }}\right)$, interframe spacing $\left(T_{\mathrm{pSIFS}}\right)$, ACK packet $\left(T_{\mathrm{ACK}}\right)$ and delay time $(\alpha)$.

$$
T=T_{\mathrm{DATA}}+T_{\mathrm{ACK}}+2 T_{\mathrm{pSIFS}}+2 \alpha
$$

Let $T_{P}, T_{\mathrm{PHY}}, T_{\mathrm{MAC}}, T_{\mathrm{BODY}}$ and $T_{\mathrm{FCS}}$ denote the duration time to transmit a preamble, PHY header, MAC header, MAC body and FCS, respectively. Therefore, the duration time to transmit a data packet is given by

$$
\begin{aligned}
T_{\mathrm{DATA}} & =T_{P}+T_{\mathrm{PHY}}+T_{\mathrm{MAC}}+T_{\mathrm{BODY}}+T_{\mathrm{FCS}}, \\
& =\frac{\text { Preamble }+ \text { PHY header }}{R_{s}}+\frac{8(\text { MAC header }+x+\text { MAC footer })}{R_{\text {hdr }}} .
\end{aligned}
$$

Since an immediate ACK/NACK carries no payload, its transmission time is represented as follows. 


$$
\begin{aligned}
T_{\mathrm{ACK}} & =T_{P}+T_{\mathrm{PHY}}+T_{\mathrm{MAC}}+T_{\mathrm{FCS}}, \\
& =\frac{\text { Preamble }+ \text { PHY header }}{R_{s}}+\frac{8(\text { MAC header }+ \text { MAC footer })}{R_{\mathrm{hdr}}} .
\end{aligned}
$$

Finally, the throughput of device $i$ is described as

$$
\begin{aligned}
\operatorname{Thro~}_{i} & =\frac{P_{\text {tran }} P_{i, \text { suc }} x r}{\left(1-P_{\text {tran }}\right) T_{s}+P_{\text {tran }} P_{\text {suc }} T+P_{\text {tran }}\left(1-P_{\text {suc }}\right) T} \\
& =\frac{P_{\text {tran }} P_{i, \text { suc }} x r}{\left(1-P_{\text {tran }}\right) T_{s}+P_{\text {tran }} T},
\end{aligned}
$$

and the system throughput becomes

$$
\text { Thro }=\sum_{i=1}^{n} \text { Thro }_{i}
$$

The throughput of scheme 2 is also represented by (12). However, several devices access the channel via the relay and the wireless LAN 1, therefore, the concentration at the wireless LAN 2 is avoided and the successful probability of all devices increases. As a result, the throughput of system is expected to increase.

\subsection{Delay}

The average access delay $D$, defined as the time elapsed between the time instant when the frame is put into service and the instant of time the frame terminates a successful delivery. Under the assumption of no retry limits, this computation is straightforward. In fact, we may rely on the well known Little's Result, which states that, for any queueing system, the average number of customers in the system is equal to the average experienced delay multiplied by the average customer departure rate. The application of Little's result to our case yields:

$$
D=\frac{x \lambda}{\frac{\text { Thro }}{n}}=\frac{x \lambda}{\text { Thro }_{i}}
$$

The delay computation is more elaborate when a frame is discarded after reaching a predetermined maximum number of retries $m$. In fact, in such a case, a correct delay computation should take into account only the frames successfully delivered at the destination, while should exclude the contribution of frames dropped because of frame retry limit (indeed, the delay experienced by dropped frames would have no practical significance).

To determine the average delay in the finite retry case, we can still start from Little's Result, but we need to replace $\lambda$ in (13) with the average number of frames that will be successfully delivered. Thus, (13) can be rewritten by

$$
D=\frac{x \lambda \beta_{i}}{\text { Thro }_{i}}
$$

here, $\beta_{i}$ denotes the probability that a randomly chosen frame will be successfully transmitted before the retransmission reaches the retry limit. Therefore, the $\beta_{i}$ is represented as follows.

$$
\beta_{i}=P_{i, \text { suc }} \sum_{j=0}^{m-1}\left(1-P_{i, \text { suc }}\right)^{j}
$$

For Scheme 2, the delay due to the multiple access at the wireless LAN 1 and 2 is similar to (14). However, the delay due to the capability of relay also should be considered. The delay due to the relay is calculated by $\frac{\sum_{j \in Q} \text { Thro }_{j}}{C}$, here

Q denote the set of devices that access the relay and the $C$ is the capability of relay. Therefore, the average delay of information data that is transmitted via relay is represented as follows. 


$$
D=\frac{x \lambda \beta_{j}}{\text { Thro }_{j}}+\frac{\sum_{j \in Q} \text { Thro }_{i}}{C}
$$

The delay of scheme 2 is the maximal delay of information data that is transmitted to wireless LAN 1 and 2.

\subsection{Bandwidth Efficiency}

In order to compare the system with and without relay, the bandwidth efficiency is adopted. The bandwidth efficiency of both schemes 1 and 2 is calculated as the ratio of total throughput of system and the total generated data. Notice that the total throughput of scheme 1 and 2 is different.

$$
\delta=\frac{\text { Thro }}{n \times \lambda} \text {. }
$$

\section{Numerical Evaluation}

The system model is the same as mentioned above and the parameters in Table 1 are used. The average distance between all devices and the wireless LAN 1 and 2 is respectively $500 \mathrm{~m}$ and $250 \mathrm{~m}$. The relay is set at halfway between the devices and the wireless LAN 1 . The delay of propagation is taken into account. The capability of relay is assumed to be $300 \mathrm{Mbps}$. The noise-free is also assumed. At first, the performance of scheme 1 is illustrated.

The throughput of scheme 1 base on lambda and the number of devices is described in Figure 5 and Figure 6, respectively. The generated data is the total data that is generated at all devices, however the generated data isn't always successfully transmitted due to the collision and the time out. Therefore, the throughput of system is considerably smaller than the generated data, especially when the number of devices and/or the lambda are high. Moreover, the delay of scheme 1 also increase when the number of devices and/or the lambda increase (Figure 7). These are the reason the scheme 2 is taken into consideration as description in Section 1.2.

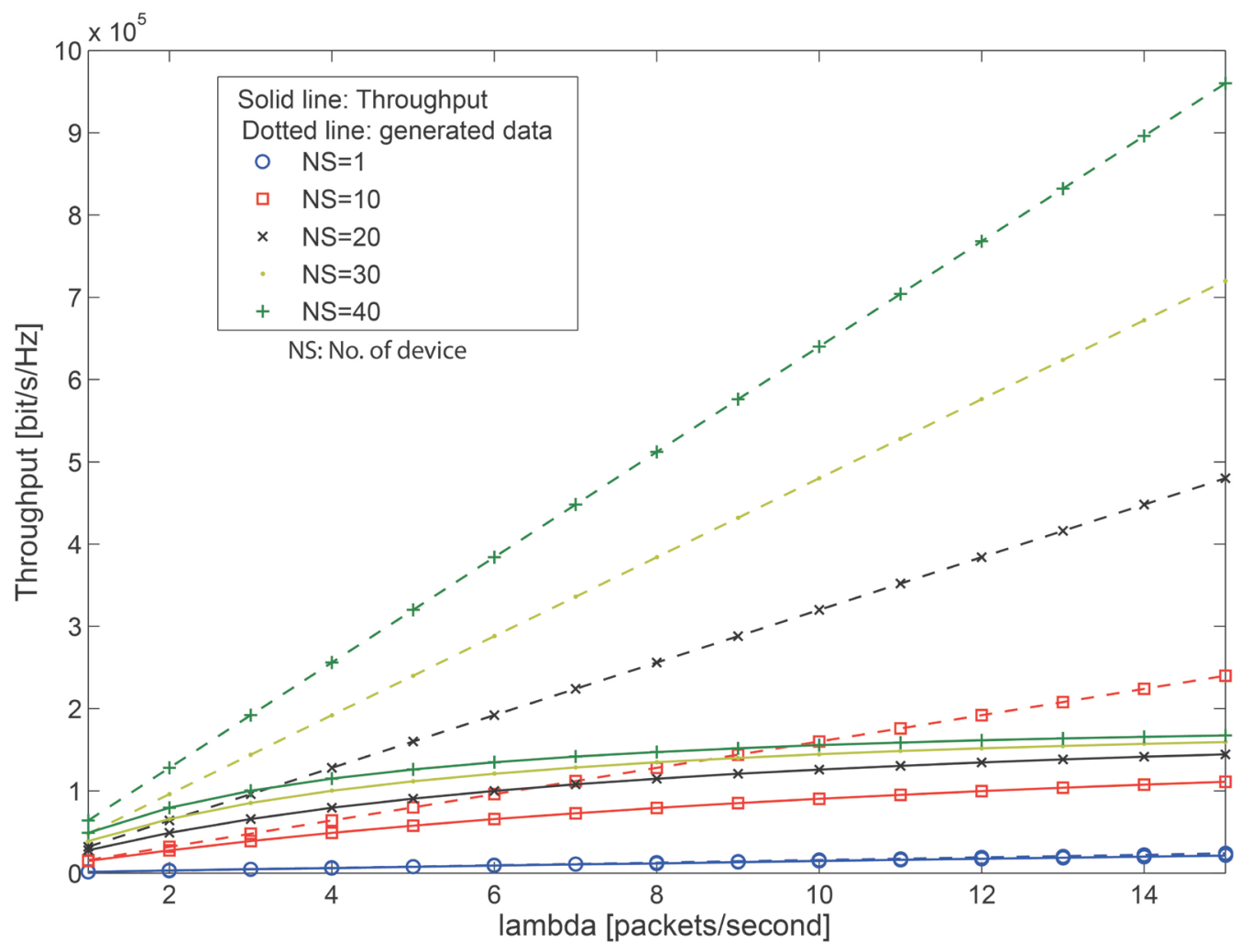

Figure 5. Throughput of scheme 1 based on lambda. 


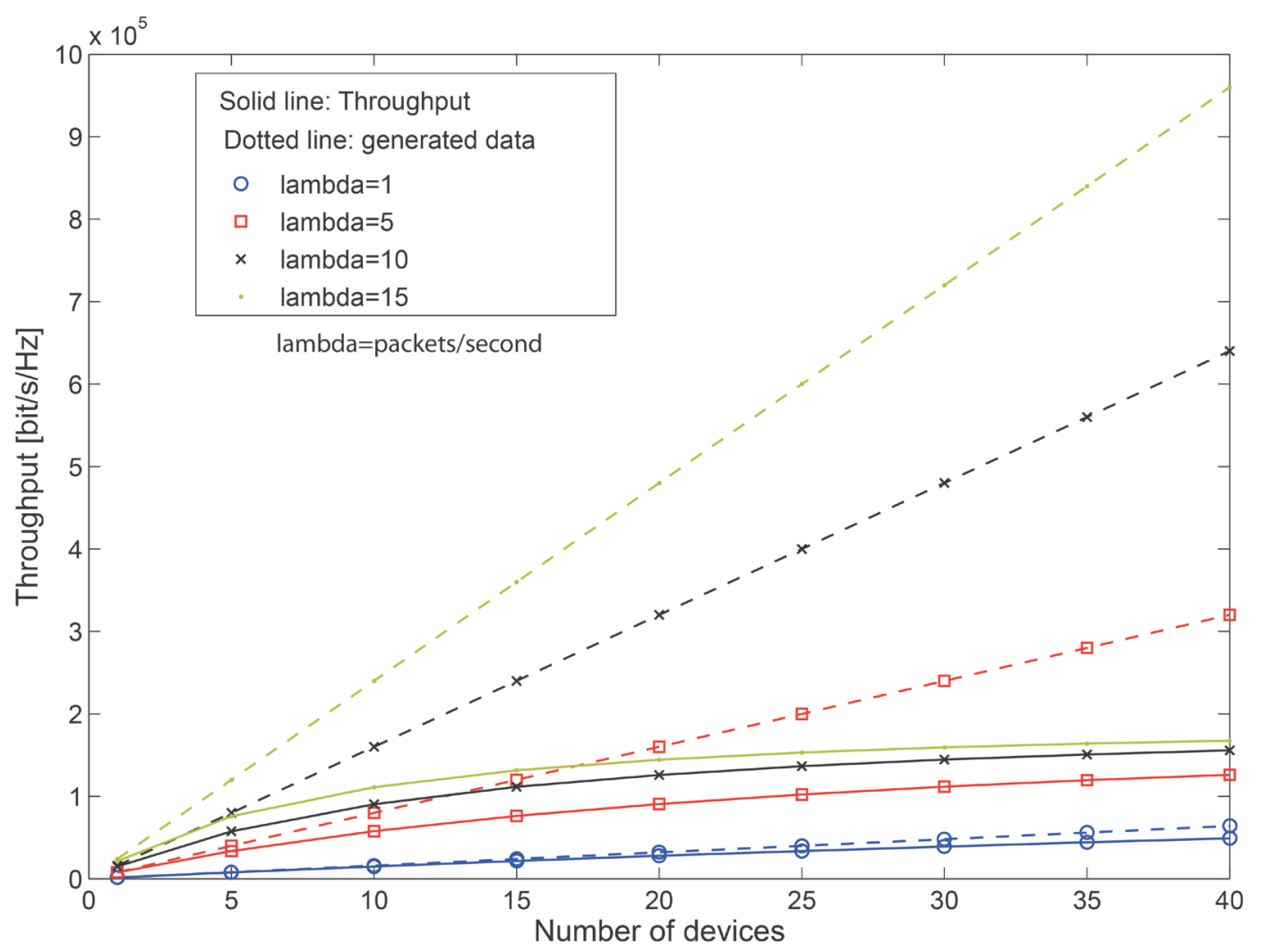

Figure 6. Throughput of scheme 1 based on the number of devices.

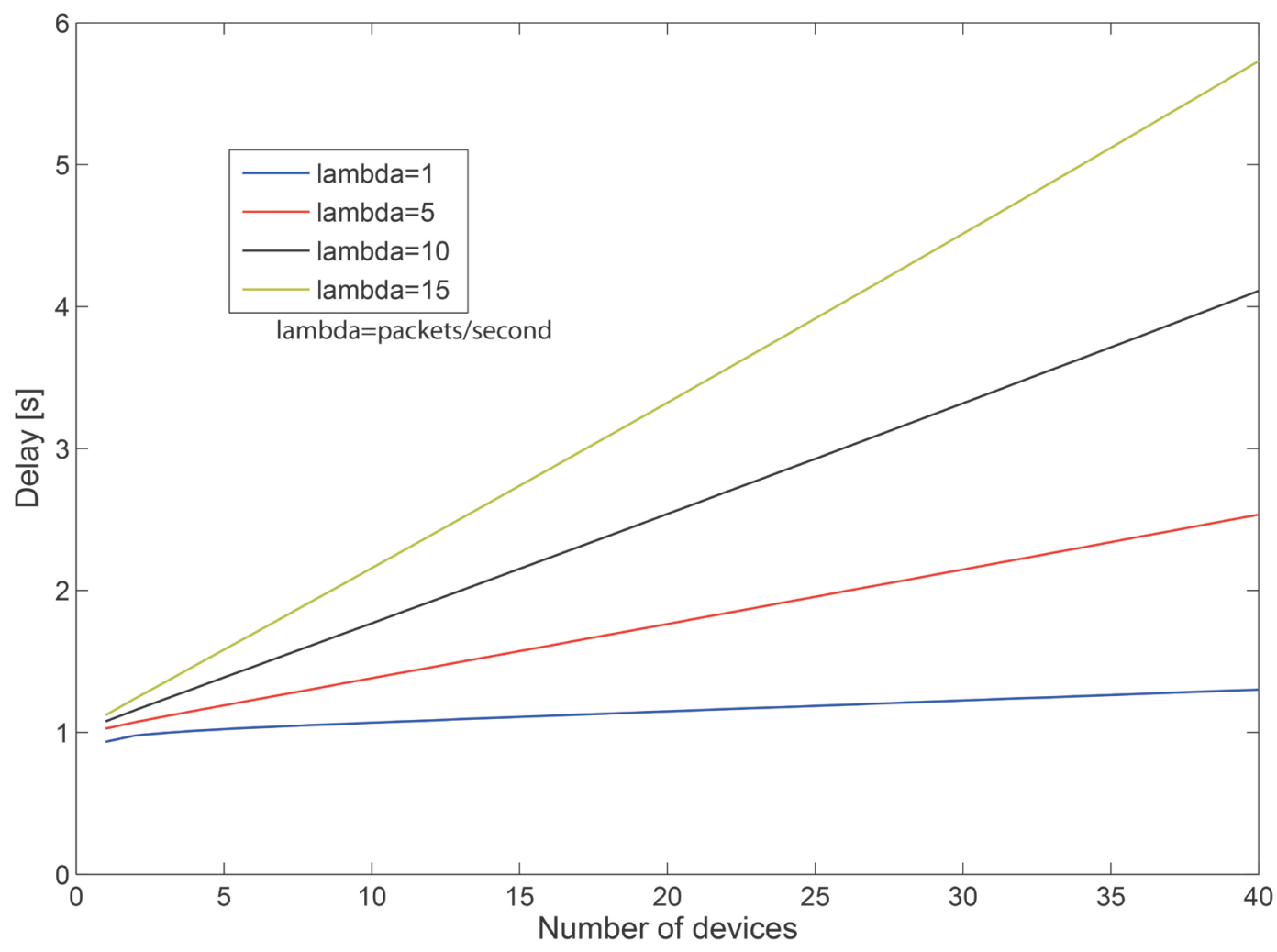

Figure 7. Delay of scheme 1. 
The comparison on the delay and the bandwidth efficiency of both schemes 1 and 2 is respectively described in Figure 8 and Figure 9, where the number of devices is fixed to be 10 and 40. For scheme 2, since the concentration at the wireless LAN 2 is avoided, the collision probability decreases. Therefore, the throughput of system increase and then the delay as well as the bandwidth efficiency increase and be higher than that of scheme 1, especially when the number of devices and/or the lambda are large. When the number of devices and the lambda are low, the difference of schemes 1 and 2 is small. Notice that the scheme 2 is more complicated due to the adding of relay and controlling the transmission of devices. It means that there are the trade off between the performance and the complication of scheme 2.

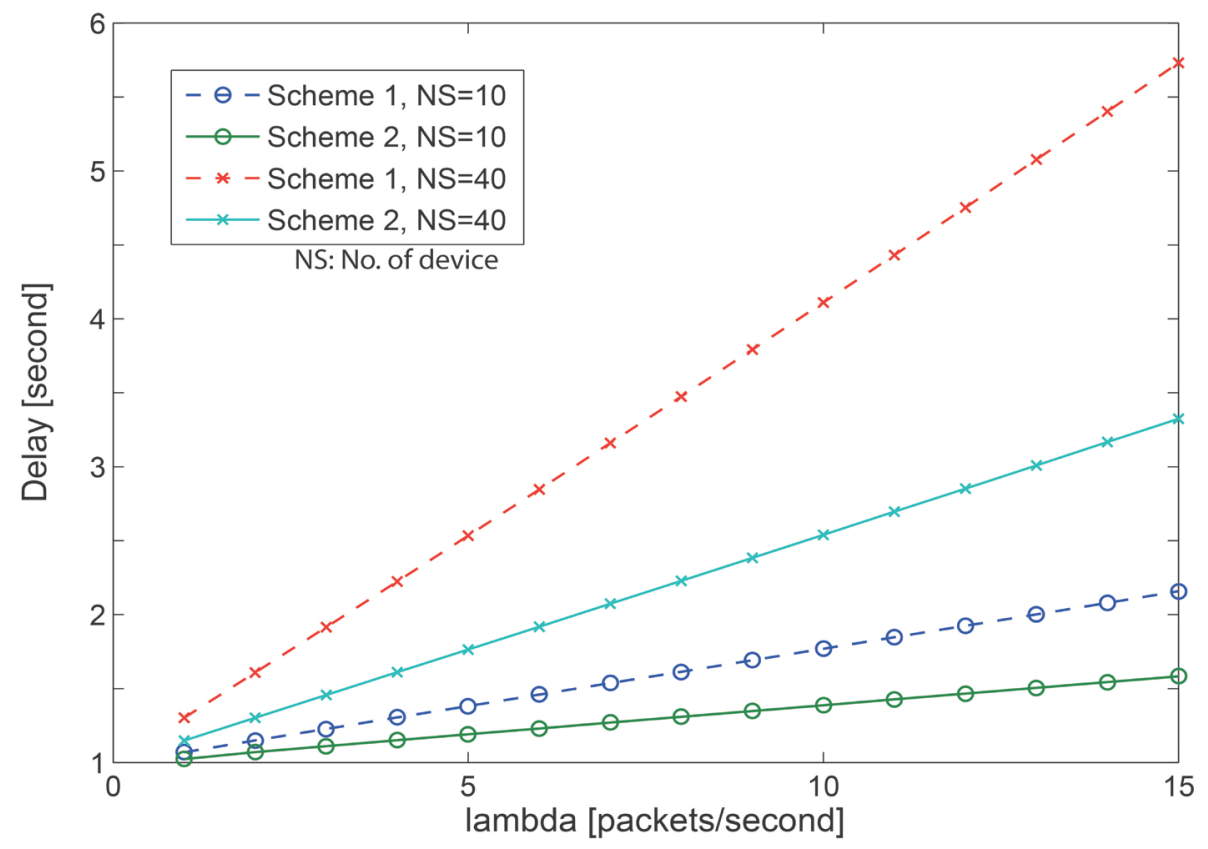

Figure 8. Delay base on lambda.

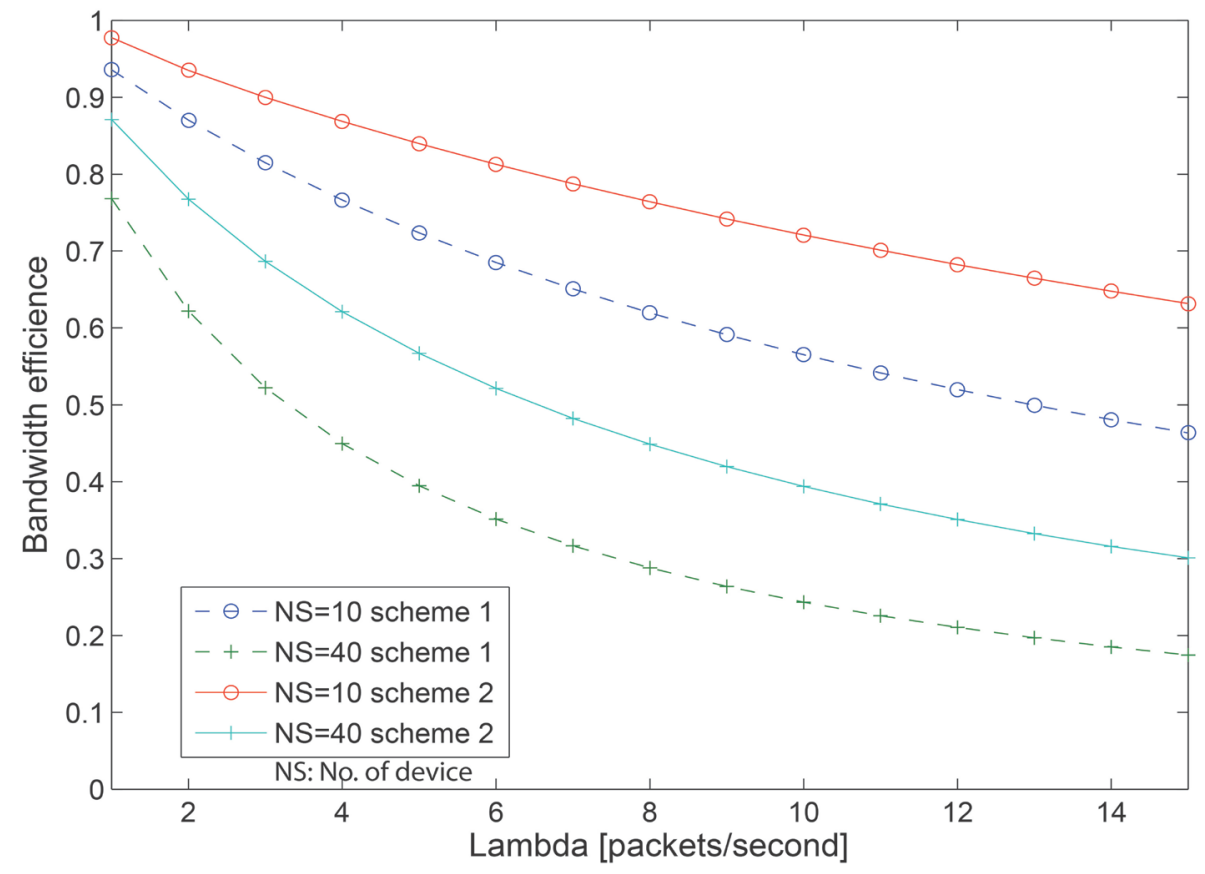

Figure 9. Bandwidth efficiency. 


\section{Conclusions}

The wireless system in a hospital has been taken into consideration and the performance is analyzed based on the standard IEEE802.15.6. The DTMC method was proposed to calculate the access probability and then the collision probability, the successful probability, the throughput, the delay, the bandwidth efficiency of system have been theoretically calculated. The performance of system with and without relay is also numerically compared, the bandwidth efficiency of scheme 2 is higher while the delay is smaller than that of scheme 1 when the number of devices and/or the packet rate are large. However, the scheme 2 is more complicated due to the controlling transmission of devices and the adding of relay.

The devices were assumed to transmit the information data to either the wireless LAN 1 or the wireless LAN 2. However, the control method hasn't been explained clearly. Moreover, the CSMA/CA was adopted, another access protocol [15] wasn't taken into account leave them to our future works.

\section{References}

[1] Wireless Personal Area Network Working Group (2012) IEEE Standard 802.15.6, Wireless Body Area Networks. IEEE Standards, 1-271.

[2] Kwak, K.S., Ullah, S. and Ullah, N. (2010) An Overview of IEEE 802.15.6 Standard. Proceedings of 3rd International Symposium on Applied Sciences in Biomedical and Communication Technologies, Rome.

[3] Martelli, F., Buratti, C. and Verdone, R. (2011) On the Performance of an IEEE 802.15.6 Wireless Body Area Network. Proceedings of European Wireless 2011, Vienna.

[4] Ullah, S., Chen, M. and Kwak, K.S. (2012) Throughput and Delay Analysis of IEEE 802.15.6-Based CSMA/CA Protocol. Journal of Medical Systems, 36, 3875-3891. http://dx.doi.org/10.1007/s10916-012-9860-0

[5] Ullah, S. and Henry Higgin, H., Braem, B., Latre, B., Blondia, C., Moerman, I., Saleem, S., Rahman, Z. and Kwak, K.S. (2012) A Comprehensive Survey of Wireless Body Area Networks on PHY, MAC, and Network Layers Solutions. Journal of Medical Systems, 36, 1065-1094. http://dx.doi.org/10.1007/s10916-010-9571-3

[6] Jung, B.H., Akbar, R.U. and Sung, D.K. (2012) Throughput, Energy Consumption, and Energy Efficiency of IEEE 802.15.6 Body Area Network (BAN) MAC Protocol. IEEE 23rd International Symposium on Personal Indoor and Mobile Radio Communications (PIMRC).

[7] Rezvani, S. and Ghorashi, A. (2012) A Novel WBAN MAC Protocol With Improved Energy Consumption and Data Rate. KSII Transactions on Internet and Information System, 6, 2302-2322.

[8] Marinkovi, S.J., Popovici, E.M., Spagnol, C., Faul, S. and Marnane, W.P. (2009) Energy-Efficient Low Duty Cycle MAC Protocol for Wireless Body Area Networks. IEEE Transactions on Information Technology in Biomedicine, 13, 915-925. http://dx.doi.org/10.1109/TITB.2009.2033591

[9] Ullah. S., An, X. and Kwak, K. (2009) Towards Power Efficient MAC Protocol for In-Body and On-Body Device Networks. Agent and Multi-Agent System: Technologies and Application, 5559, 335-345.

[10] Zhen, B., Li, H.B. and Kohno, R. (2008) IEEE Body Area Networks and Medical Implant Communications. Proceedings of the ICST 3rd International Conference on Body Area Networks, Tempe.

[11] Rashwand, S., Misic, J. and Khazaei, H. (2011) IEEE 802.15.6 under Saturation: Some Problems to be Expected. Journal of Communications and Networks, 13, 142-148. http://dx.doi.org/10.1109/JCN.2011.6157413

[12] Rashwand, S. and Misic, J. (2011) Performance Evaluation of IEEE 802.15.6 under Non-Saturation Condition. Proceedings of IEEE Global Telecommunications Conference (GLOBECOM), Kathmandu.

[13] Li, C.L., Geng, X., Yuan, J. and Sun, T. (2013) Performance Analysis of IEEE 802.15.6 MAC Protocol in Beacon Mode with Superframes. KSII Transaction on Internet and Information Systems, 7, 1108-1130. http://dx.doi.org/10.3837/tiis.2013.05.010

[14] Rashwand, S. and Misic, J. (2012) Effects of Access Phases Lengths on Performance of IEEE 802.15.6 CSMA/CA. Journal of Computer Networks, 56, 2832-2846. http://dx.doi.org/10.1016/j.comnet.2012.04.023

[15] Bianchi, G. (2000) Performance Analysis of the IEEE 802.11 Distributed Coordination Function. IEEE Journal on Selected Areas in Communications, 18, 535-547. http://dx.doi.org/10.1109/49.840210 
Scientific Research Publishing (SCIRP) is one of the largest Open Access journal publishers. It is currently publishing more than 200 open access, online, peer-reviewed journals covering a wide range of academic disciplines. SCIRP serves the worldwide academic communities and contributes to the progress and application of science with its publication.

Other selected journals from SCIRP are listed as below. Submit your manuscript to us via either submit@scirp.org or Online Submission Portal.
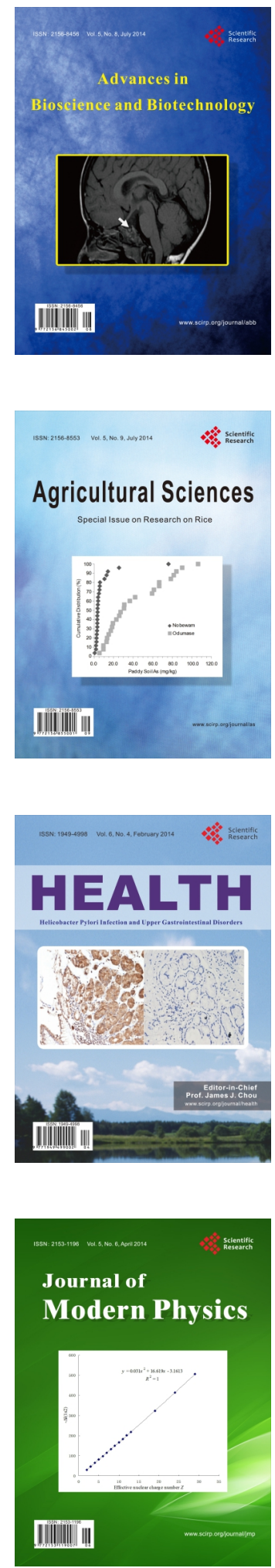
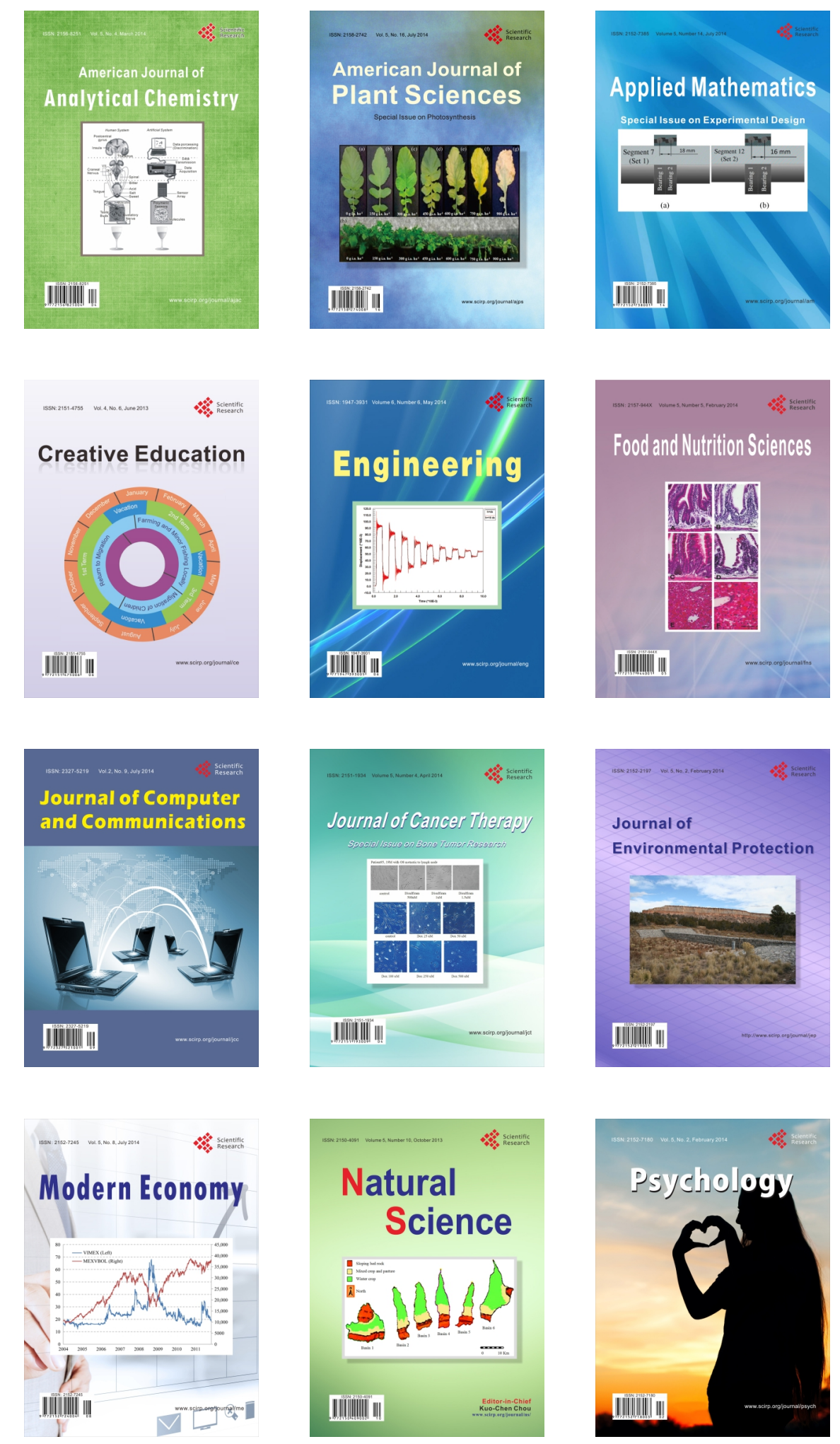